\title{
Asymmetry of lower limb strength and jumping ability of young soccer players
}

\author{
ALICJA RUTKOWSKA-KUCHARSKA* \\ ${ }^{1}$ University School of Physical Education in Wrocław, Department of Biomechanics, Wrocław, Poland.
}

\begin{abstract}
Purpose: The aim of this study was to evaluate the potential correlation between asymmetry of lower limb muscle torque, asymmetry of vertical ground reaction force during take-off in young soccer players and their jumping abilities. Methods: Twenty-three young soccer player $(16.9 \pm 0.64$ years old) participated in measurements. An isokinetic dynamometer, the Biodex System, was applied to test muscle torque (PT) of the knee flexors and extensors. The vertical ground reaction force (vGRF) was recorded from two Kistler plates. Jumping abilities were assessed with the horizontal (HJ) and vertical jump (VJ) tests. The asymmetry index (AI) was used to assess the asymmetry of the limbs. Results: The asymmetry index showed the highest asymmetry (over 10\%) for the PT under static conditions for knee flexors and extensors. The correlation $(-0.432, p=0.038)$ was found between the asymmetry of vGRF and the height of the VJ. There was no correlation between the muscle torque and the height of the vertical jump. However, a correlation between the HJ length and muscle torque for flexors and extensors of the right and left lower limb was found. Conclusions: The asymmetry of the muscle torque of the flexors and extensors of the knee joint does not correlate with the results of both jumping ability tests. There was a statistically significant correlation between the vGRF asymmetry index during take-off and the height of the VJ. In the HJ, such a relationship was not found.
\end{abstract}

Key words: muscle torque, GRF, jumping tests

\section{Introduction}

Many researchers have investigated the problem of muscle strength symmetry in sports. The issue has been mainly addressed by those who are interested in movement asymmetry. It is also reported that asymmetry of muscle strength results in injuries to muscle groups directly involved in movements specific to sports activities [16], [22], but can also cause lower back pain [21]. Rodriquez-Lorenzo et al. [18], [19] explained the need for symmetry in the lower limbs of soccer players. Their study proved that strength training equal in both legs minimises the potential asymmetry of forces acting on joints, reduces muscle imbalances and decreases the workload on the preferred leg, which may eventually lead to injuries. This researcher, referring to the papers of other authors, points out the relationship between the strength of lower limbs and kicking ability manifested by maximum kicking velocity. Therefore, improving the maximum kicking velocity with both the preferred and non-preferred leg must be an important objective for soccer coaches. Knee extensors in football players are important in kicking, cutting skills or jumping to head the ball [3]. They also stabilize knee joint during cuts and slide tackles. These skills are clearly unilateral and require asymmetric movement patterns so they develop asymmetric adaptations of the musculoskeletal functions in the lower limbs [7]. As claimed by many researchers, one of the causes of injuries in soccer players is the asymmetry of strength between the dominant and non-dominant limb. Therefore, the training of young players should focus on the symmetrical development of the strength of the right and left the lower side of the body [26]. Daneshjoo et al. [5] indicated the possibility that over time the unique muscle-loading patterns experienced in soccer, to-

\footnotetext{
* Corresponding author: Alicja Rutkowska-Kucharska, Department of Biomechanics, University School of Physical Education in Wrocław, al. I.J. Paderewskiego 35, 51-612, Wrocław, Poland. Phone: +48605623399, e-mail: alicja.rutkowska-kucharska@awf.wroc.pl Received: November 26th, 2019

Accepted for publication: January 21st, 2020
} 
gether with the superior use of the dominant leg, may result in a bilateral asymmetrical increase in the concentric strength of the quadriceps in the dominant kicking leg.

It is common for coaches to use jumping tests such as the vertical and horizontal jump to assess the strength of lower limbs in young athletes. The tests evaluate the strength of lower limb extensors in the knee and hip joints. Coordination movements of body segments during take-off in the vertical jump and horizontal jump are different. The ground reaction force vector during the vertical jump is directed vertically upwards, and in the horizontal jump, forward at an angle of approx. $45^{\circ}$. Moreover, Nagano and coauthors [15] in their simulation study showed that the profiles of the optimal muscle activation were markedly different between the horizontal and the vertical jump. The results of both tests depend not only on the strength of the lower limbs but also on take-off technique, which consists of the ability to coordinate the movement of the arms with the rest of the body and depth of squatting [8]. The take-off for both jumps requires muscle strength in both lower limbs. This means that the asymmetry of the muscle strength generated in the take-off phase can affect the results of both tests.

Jumping exercises performed with take-off from both legs in different directions are popular exercises in training because they symmetrically develop strength. In the case of young athletes, the strength of right and left body muscles is important in injury-prevention training. The symmetry of force generated during take-off in different types of jumps can be assumed to have an influence on the performance of a competitor. In their studies, Fousekis et al. [7] demonstrated the importance of this problem in the case of young soccer players. They found that soccer players with intermediate to early professional skills showed a tendency for greater isokinetic strength asymmetries than players with more professional experience.

The aim of this study was to evaluate the potential correlation between asymmetry of lower limb muscle torque, asymmetry of vertical ground reaction force during take-off in young soccer players and their jumping abilities. It has been assumed that the symmetry of muscle torque in knee flexors and extensors may be related to the results of jumping tests. Takeoff in the jumping tests takes place with both limbs simultaneously; thus the study also assessed the possible correlation between the symmetry of the vertical component of the ground reaction force in the take-off phase in jumping tests and their results. To our knowledge, no previous studies have examined this dependency.

\section{Materials and methods}

\subsection{Participants}

The research group comprised twenty-three soccer players - with a minimum of four years' training experience - playing in the Central Junior League, which is the highest level of competition in Poland under age 19. Their mean age was $16.9 \pm 0.64$ years, body height $1.78 \pm 0.055 \mathrm{~m}$, body mass $70.9 \pm 5.6 \mathrm{~kg}$. A survey carried out prior to measurements showed that the players indicated their right lower limb as dominant in playing soccer. To determine the dominant limb, subjects were asked which limb they usually use to do the penalty shot. They did not suffer from any injuries at least 6 months prior to testing and signed a health declaration, which enabled them to perform testing with maximum loading. All the participants expressed informed consent to participate in the experiment. In the case of minors, consent was expressed by their guardians. All the participants were informed about the aim and methodology of the study. The study was conducted according to the principles expressed in the Declaration of Helsinki and received the approval of the Senate Ethics Committee of our university. All the measurements were carried out in the Biomechanics Analysis Laboratory (PN-EN ISO 9001:2001).

\subsection{Measurement procedure}

The tests were carried out during the transitional period that occurs before the beginning of preparations for the spring season. The measurements were preceded by a 5-minute warm-up including a run, two and single-leg jumps and squats. Each athlete performed the tests randomly in order to eliminate the effect of fatigue on the results.

\section{Torque measurements}

An isokinetic dynamometer, Biodex System 4 Pro., was applied to test peak muscle torque (PT) in knee flexors and extensors under isometric and isokinetic conditions. The measurements were taken in accordance with the measurement procedure recommended by the manufacturer of Biodex System 4 Pro. The measurement setup consisted of a chair with an adjustable back angle and seat height, and straps to stabilize the trunk (2 straps) and pelvis (1 strap). All individuals were allowed to familiarize themselves with the type and 
resistance of movement to be performed. The design of the device enabled the alignment of the dynamometer axis of rotation with the axis of the kneejoint movement. All measurements were taken in the sagittal plane under isokinetic (angular velocity $30 \%$, $60 \%$ and $120 \%$ s) and isometric conditions $(0 \% \mathrm{~s})$. The subject performed five cycles of maximum knee flexion under isometric conditions with the lower right and left limb at a $30^{\circ}$ flexion position, and the extension, with the limb at a $75^{\circ}$ position at the knee joint. The measurement under isokinetic conditions was also performed in five motion cycles too. The range of knee flexion and extension was this same as in isometric measurements. Out of the five cycles, the first and the last were excluded from statistical calculations. The sequence of measurements, for each limb and each measurement, was random.

\section{Ground reaction force}

Vertical ground reaction force (vGRF) data were collected using two Kistler 9286AA-A plates with the frequency of $1 \mathrm{kHz}$. To carry out the jumping ability tests, a subject took a standing erect position on two parallel Kistler plates. Each athlete, in both jumping ability tests, performed three jumps. The vertical ground reaction force (vGRF) was recorded for each limb during the take-off phase in the horizontal (HJ) and vertical jump (VJ). Also, time of flight was recorded for each limb in order to calculate the height of the vertical jump (from the moment of take-off from the Kistler plate to contact of the feet with the plate).

\section{Horizontal jump test}

An athlete stood with both feet on the Kistler plates on a line, and upon a signal performed the longest possible jump, employing an arm swing to enhance performance. There was a one-minute interval between individual samples. The longest jump was selected out of the three samples for maximum vGRF analysis and statistical calculations. Jump length was measured from the take-off line to the point of contact between the heel and the ground. The accuracy of the length measured was to the nearest $1 \mathrm{~cm}$.

\section{Vertical jump test}

As in the previous test, the competitor stood with each foot on separate Kistler plates and upon a signal performed a vertical jump with the highest possible arm swing. The Bosco formula [2] was used to calculate the height of the jump for all three samples of flight time. Time from take-off to landing was calculated for each limb separately. The final result of the jump height was calculated as the mean of both limbs. For statistical calculations, the highest value of height was selected from the three samples.

\subsection{Assessment of symmetry}

The following formula (1) was applied to assess the asymmetry of muscle torque of knee flexors and extensors and asymmetry of vGRF during take-off in the jumping tests:

$$
\mathrm{AI}=\frac{\left|x_{l}-x_{r}\right|}{0.5 \cdot\left(x_{l}+x_{r}\right)} \cdot 100 \%
$$

$\mathrm{AI}$ - asymmetry index, $x$ - variable, $l-$ left limb, $r$ - right limb.

\subsection{Statistical analysis}

Prior to statistical calculations, the vGRF values and the muscle torque of knee flexors and extensors were normalized to the body mass of each athlete. The distribution of all variables investigated was evaluated by the Kolmogorov-Smirnov test and the Shapiro-Wilk test. None of the variables had a normal distribution. Spearman's rank correlation coefficient was used to evaluate correlations between all parameters. For the statistical analyses, the value of $\alpha=0.05$ was considered significant. All computations were performed with STATISTICA software.

\section{Results}

Analysis of the asymmetry index (AI) of the variables studied (Table 1) showed the highest asymmetry (over 10\%) between the lower limbs for the measurement of muscle torque (PT) under static conditions for both knee flexors and extensors. The mean values in the remaining measurement conditions were within normal limits (AI under 10\%). However, the values of the standard deviation of PT and VGRF showed an increase in individual cases of AI (over 15\%). This included asymmetry of muscle torque (PT) in knee flexors at $30^{\circ} / \mathrm{s}$ and vGRF asymmetry during the takeoff of horizontal jumps (HJ).

The aim of the study was to test the hypothesis that the results of jumping tests may be related to the symmetry of muscle torque of the lower limb. The asymmetry of the muscle torque of the flexors and 
Table 1. Mean values of variables describing the strength of the right $(\mathrm{R})$ and left $(\mathrm{L})$ leg and asymmetry index. $\mathrm{PT}$ - peak torque. vGRF - vertical ground reaction force

\begin{tabular}{|c|c|c|c|c|c|}
\hline \multirow[b]{2}{*}{ Variables } & \multicolumn{2}{|c|}{ Dominant leg (R) } & \multicolumn{2}{|c|}{ Non-dominant leg (L) } & \multirow{2}{*}{$\frac{\text { Asymmetry Index [\%] }}{\text { Mean } \pm \mathrm{SD}}$} \\
\hline & Mean \pm SD & $\begin{array}{c}\text { Coefficient } \\
\text { of variation [\%] }\end{array}$ & Mean \pm SD & $\begin{array}{c}\text { Coefficient } \\
\text { of variation [\%] }\end{array}$ & \\
\hline PT [Nm/kg] & \multicolumn{5}{|c|}{ Flexors } \\
\hline \begin{tabular}{l|l} 
& $0 \% / \mathrm{s}$
\end{tabular} & $2.35 \pm 0.246$ & 10.22 & $2.18 \pm 0.025$ & 11.56 & $11.40 \pm 7.84$ \\
\hline $30 \% \mathrm{~s}$ & $2.25 \pm 0.386$ & 16.91 & $2.11 \pm 0.027$ & 12.85 & $9.01 \pm 10.89$ \\
\hline $60 \%$ & $2.15 \pm 0.200$ & 9.33 & $2.01 \pm 0.021$ & 10.28 & $7.96 \pm 5.31$ \\
\hline $120 \%$ & $1.90 \pm 0.191$ & 10.06 & $1.81 \pm 0.023$ & 12.50 & $8.41 \pm 6.66$ \\
\hline \multicolumn{6}{|c|}{ Extensors } \\
\hline $0 \% \mathrm{~s}$ & $3.64 \pm 0.496$ & 13.63 & $3.84 \pm 0.069$ & 18.04 & $10.50 \pm 8.32$ \\
\hline $30 \% \mathrm{~s}$ & $3.43 \pm 0.492$ & 14.38 & $3.48 \pm 0.060$ & 17.29 & $7.80 \pm 4.60$ \\
\hline $60 \%$ & $3.16 \pm 0.371$ & 11.75 & $3.13 \pm 0.042$ & 13.57 & $5.89 \pm 4.17$ \\
\hline $120 \% \mathrm{~s}$ & $2.56 \pm 0.316$ & 12.32 & $2.51 \pm 0.033$ & 12.84 & $6.75 \pm 5.55$ \\
\hline \multicolumn{6}{|c|}{ Vertical jump (VJ) } \\
\hline vGRF [N/kg] & $12.15 \pm 1.288$ & 10.60 & $12.11 \pm 1.111$ & 9.18 & $8.16 \pm 5.44$ \\
\hline \multicolumn{6}{|c|}{ Horizontal jump (HJ) } \\
\hline vGRF [N/kg] & $11.49 \pm 2.691$ & 23.40 & $11.43 \pm 2.115$ & 18.49 & $8.69 \pm 7.03$ \\
\hline
\end{tabular}

Table 2. Spearman's correlations (only statistically significant) matrix between the length of horizontal jump and muscle torque (PT) and vertical ground reaction force (vGRF) for the right (R) and left (L) leg

\begin{tabular}{|c|c|c|c|c|c|c|c|}
\hline \multirow{2}{*}{ Variables } & \multirow{2}{*}{ Muscles } & \multirow{2}{*}{ Leg } & \multicolumn{5}{|c|}{ Correlation coefficient } \\
\cline { 4 - 8 } & & & $0 \% \mathrm{~s}$ & $30 \% \mathrm{~s}$ & $60 \% \mathrm{~s}$ & $120 \% \mathrm{~s}$ & \\
\hline PT & & & & & & & \\
\hline & Extensors & & & & & & \\
\hline & & $\mathrm{R}$ & 0.434 & 0.430 & 0.528 & 0.549 & \\
\hline & & $\mathrm{L}$ & 0.483 & - & - & 0.527 & \\
\hline & Flexors & & & & & & \\
\hline & & $\mathrm{R}$ & - & 0.460 & 0.532 & - & \\
\hline & & $\mathrm{L}$ & 0.455 & 0.542 & 0.537 & - & \\
\hline & & & & & & & \\
\hline vGRF & & $\mathrm{L}$ & & & & & 0.456 \\
\hline
\end{tabular}

Table 3. Mean value, standard deviation, variation and correlation coefficient between the height of VJ and length of HJ

\begin{tabular}{|l|c|c|c|c|}
\hline $\begin{array}{c}\text { Jumping } \\
\text { ability tests }\end{array}$ & Mean \pm SD & Min-max & $\begin{array}{c}\text { Coefficient } \\
\text { of variation [\%] }\end{array}$ & $\begin{array}{c}\text { Correlation } \\
\text { coefficient }\end{array}$ \\
\cline { 1 - 4 } Vertical jump $[\mathrm{m}]$ & $0.331 \pm 0.029$ & $0.28-0.40$ & 8.61 & \multirow{2}{*}{0.123} \\
\hline Horizontal jump $[\mathrm{m}]$ & $2.369 \pm 12.86$ & $2.17-2.63$ & 5.43 & \\
\hline
\end{tabular}

extensors of the knee joint does not correlate with the results of jumping ability tests. It is interesting to note that there was no statistically significant correlation between muscle torque knee flexors and extensors under isometric and isokinetic conditions for the height of the vertical jump (Table 2). On the other hand, a statistically significant correlation between the length of a horizontal jump and muscle torque for flexors and extensors of the right and left lower limb was found in many cases for HJ.
The next aim of the study was to examine the relationship between the asymmetry of lower limb muscle strength generated in the take-off phase and the results of the jumping ability tests. Statistical analysis showed a statistically significant correlation $(-0.432$, $p=0.038$ ) between asymmetry index of vGRF during take-off and the height of the vertical jump. It means that the smaller the asymmetry of the VGRF, the higher is the vertical jump. There was no such correlation for length of the horizontal jump. Moreover, the 
analysis showed the relationship between vGRF of the left lower limb and jump length found for the HJ test, while the height of the VJ was statistically significant $(0.605, p=0.006)$ for the vGRF of the right limb (Table 2)

Since the relationship between the muscle torque of the lower limb and the results of the jumping tests were different for both tests, the correlation between the results of both tests was examined. There was no statistically significant association between the HJ length and the VJ height (Table 3).

\section{Discussion}

Authors investigating the causes of lower limb injuries in soccer mainly focus on the incorrect PT ratio of flexors to extensors in the knee joint [3], [8], [17], [24]. Thus, there are not many studies which have aimed to assess the asymmetry of strength between the lower limbs of soccer players with regard to injuries. As mentioned in the introduction, each player prefers either the right or the left limb during the game. In the case of young competitors with short training experience, such conditions may cause a significant load on one of the lower limbs which can lead to an injury. With this in mind, this study aimed to assess the asymmetry in the lower limbs under isokinetic conditions and during the performance of a motor task similar in structure to the exercises shaping the jumping abilities of a soccer player. The tested players participated in the same training programme; therefore, they were a homogeneous group. They presented similar skills, but the relative difference in training experience suggested that the participants may have different strength capabilities in the lower limbs. The level of jumping abilities in the examined athletes evaluated by the height of a vertical jump with an arm swing was lower than athletes from national teams tested by Turner et al. [24] $(45.1 \pm 1.7 \mathrm{~cm})$, Chamari [4] $(62 \pm 5.6 \mathrm{~cm})$, and Yanci [25] (48.86 $\pm 5.71 \mathrm{~cm})$, but higher than those of physical education university students [14] $(23.15 \pm 3.66 \mathrm{~cm})$. On the other hand, the length of the horizontal jump of the examined players was greater than that of professional soccer players $(2.39 \pm 0.14 \mathrm{~m})$ examined by Yanci and co-authors [25].

The asymmetry index (AI) exceeding $10 \%$ is considered by many researchers to be the deficit of strength that can contribute to the knee risk factor [1], [5], [7] Also in this study, the researchers assumed the value of $10 \%$ asymmetry index as a safe value for players. It is interesting that Hoffman et al. [11] found in their study that such an asymmetry in lower limb power does not appear to relate to performance differences during direction-specific agility tests. Malý [12], when researching young soccer players, did not find any differences in muscle torque between preferred and non-preferred limb. Zakas [26], when studying elite soccer players, did not find asymmetry between muscle torque of lower limbs. This was explained by the fact that training sessions and matches appear to have imposed a strength balance for the right and left body sides of professional soccer players. In this study, some variables were characterized by an asymmetry exceeding $10 \%$. The highest asymmetry was reported between the lower limbs for the measurement of muscle torque (PT) under static conditions for both knee flexors and extensors. Although the mean values of the tested group in the remaining measurement conditions were within normal limits (AI below 10\%), the values of the standard deviation of peak torque (PT) and ground reaction force (vGRF) indicated an increase in individual cases of AI (above 15\%). This includes asymmetry of muscle torque in knee flexors at $30^{\circ} / \mathrm{s}$ and vGRF asymmetry during the take-off of a horizontal jump (HJ). In our opinion, this AI value may indicate an increased risk of injury in these players in the future. This is confirmed by much research. It would seem magnitudes of $15 \%$ or more are often associated with players who have recently sustained an injury, whereas magnitudes below $10 \%$ are typically reported in non-injured populations. A threshold of $15 \%$ can be used to identify players who may be at risk of incurring a lower limb injury and, therefore, requiring additional training focused on correcting the limb asymmetry in the appropriate manner [10], [16], [22].

The main problem of our research was related to the relationship between the asymmetry of muscle strength in lower limbs and the jumping abilities of soccer players. The asymmetry of the muscle torque of the flexors and extensors of the knee joint does not correlate with the results of both jumping ability tests. However, a statistically significant correlation was found between the asymmetry of vGRF and the height of the vertical jump. This problem was taken up because it is common for coaches to assume that the results of jumping tests depend on the strength and power of the lower limbs. Therefore, we presumed that a smaller asymmetry of vGRF during takeoff will lead to better jumping results. Yanci et al. [25], in their studies on the symmetry of length and height of single-legged jumps, found slight differences be- 
tween the results for the right and left limb (from 0.63 to $4.04 \%$ ). Similar results were obtained by Maulder [13], who verified the asymmetry index in jumping tests in healthy young men and observed very little difference between dominant and non-dominant legs. In the case of our soccer players, there was a statistically significant correlation $(-0.432, p=0.038)$ between the vGRF asymmetry index during take-off and the height of the VJ. In the HJ, such a relationship was not found.

There have been numerous studies that have investigated the relationship between muscle torque and physical fitness tests, including the jumping tests used in this study [4], [6], [9], [20]. The results of our study indicated a relationship between PT (isometric conditions and $120 \%$ s) extensors and flexors $(30 \%$ and $60 \%$ s) of both lower limbs and $\mathrm{HJ}$ length. However, this study did not reveal any correlation between the $\mathrm{VJ}$ height and muscle torque (PT) measurement. The relationship between VJ height and PT $(60 \%$ and $180 \%$ s) in collegiate soccer players was reported by Hamilton [9]. Also RodríguezLorenzo [18] confirmed the link between peak torque of right and left leg knee flexor muscles, evaluated at $60 \%$ speed with countermovement jump. In a study on professional striker soccer players, Buśko et al. [3] looked for a link between peak muscle torque (PT) and the height of akimbo countermovement (ACMJ), countermovement (CMJ) and spike (SPJ) jumps. Their results demonstrated a relationship between maximum muscle torque in knee extensors developed during the isokinetic contraction of three types of jumps. Jump height correlated with isokinetic muscle strength in the knee extensor, but not with muscle torques developed in isometric contraction. Dobbs [6], looking for a relationship between kinetic and kinematic variables measured in the horizontal jump, found stronger correlations with sprint speed than vertical jump height. Śliwowski [23] found no significant correlations between any of the jumping tests and peak torque of knee extensors of both legs at $60 \%$ s. The results obtained in our study revealed an interesting fact, namely the relationship between the length of the HJ and the vGRF. The higher the value of the vGRF was, the longer was the jump. The lack of unequivocal results indicating a relationship between laboratory measurements and jumping tests, especially in the case of young athletes, may be due to the fact that jumping abilities, apart from the strength and power of the lower limbs, is determined by other factors. This may be related to the ability to coordinate movements of body segments.

\section{Conclusions}

Our findings and those of other authors indicate the necessity to perform laboratory measurements evaluating the asymmetry of strength in injury prevention. Such measurements cannot be replaced by jumping tests. That laboratory tests evaluating the strength of soccer players are better at diagnostics. It should be kept in mind that the strength capabilities of a player are one of the building blocks of success in this sport.

In the case of these soccer players, the average values of the asymmetry index (AI), muscle torque (PT) and ground reaction force (vGRF) did not exceed $10 \%$. However, the values of standard deviation in individual cases of AI were significantly higher than $15 \%$. This applied especially to the asymmetry of PT $30 \%$ in knee flexors. This means that the assessment of the asymmetry of the strength of the lower limbs is useful primarily in the prevention of injury.

\section{Acknowledgments}

Thank you mgr Wojciech Łopusiewicz for assistance in preparing the measurement data for statistical analysis.

\section{References}

[1] Bishop CH., Read P., Shyam CH., Turner A., Asymmetries of the lower limb: the calculation conundrum in strength training and conditioning, Strength. Cond. J., 2016, 38(6), 27-32, DOI: 10.1519/SSC.0000000000000264.

[2] Bosco C., Luhtanen P., Komi P.V., A simple method for measurement of mechanical power in jumping, Eur. J. Appl. Physiol., 1983, 50 (2), 273-282.

[3] BuŚKO K., GÓRSKi M., NiKOlAidis T.P., MAZUR-RÓŻYCKA J., ŁACH P., STANIAK Z., GAJEWSKI J., Leg strength and power in polish striker soccer players, Acta Bioeng. Biomech., 2018, 20 (2), 109-116, DOI: 10.5277/abb-01066-2017-02.

[4] Chamari K., Chaouachi A., Hambli M., Kaouech F., WisløfF U., Castagna C., The five-jump test for distance as a field test to assess lower limb explosive power in soccer players, J. Strength Cond. Res., 2008, 22 (3), 944-950, DOI: 10.1519/JSC.0b013e31816a57c6.

[5] Daneshjoo A., Nader Rahnama N., Halim Mokhtar A., YUSOF A., Bilateral and unilateral asymmetries of isokinetic strength and flexibility in male young professional soccer players, J. Hum. Kinet., 2013, 36, 45-53, DOI: 10.2478/hukin-2013-0005 45.

[6] Dobbs C.W., Gill N.D., Smart D.J., McGuigan M.R., Relationship between vertical and horizontal jump variables and muscular performance in athletes, J. Strength. Cond. Res., 2015, 29 (3), 661-671, DOI: 10.1519/JSC.0000000000000694.

[7] FouseKis K., TsePis E., VAGENAS G., Lower limb strength in professional soccer players: profile, asymmetry, and training age, J. Sports Sci. Med., 2010, 9 (3), 364-73. 
[8] Gajewski J., Michalski R., BuŚKo K., MaZur-RóżycKa J., STANIAK Z., Countermovement depth - a variable which clarifies the relationship between the maximum power output and height of a vertical jump, Acta Bioeng. Biomech., 2018, 20 (1), 127-134, DOI: 10.5277/ABB-01058-2017-02.

[9] Hamilton R.T., Shultz S.J., Schmitz R.J., Perrin D.H., Triple-Hop distance as a valid predictor of lower limb strength and power, J. Athl. Train., 2008, 43 (2), 144-151.

[10] Hewit J., Cronin J., Hume P., Multidirectional leg asymmetry assessment in sport, Strength Cond. J., 2012, 34 (1), 83-86, DOI: $10.1519 /$ SSC.0b013e31823e $83 \mathrm{db}$.

[11] Hoffman J., Ratamess N., Klatt M., Faigenbaum A., KANG J., Do bilateral power deficits influence direction-specific movement patterns?, Res. Sports Med., 2007, 15, 125-132, DOI: $10.1080 / 15438620701405313$.

[12] MALÝ T., ZAHÁLKA F., MALÁ L., Isokinetic strength, ipsilateral and bilateral ratio of peak muscle torque in knee flexors and extensors in elite young soccer players, Acta Kinesiologica, 2010, 4 (2), 17-23.

[13] Maulder P., Cronin J., Horizontal and vertical jump assessment: reliability, symmetry, discriminative and predictive ability, Physical Therapy in Sport, 2005, 6, 74-82, DOI: 10.1016/j.ptsp.2005.01.001.

[14] Meylan C., McMaster T., Cronin J., Mohammad N.I., Rogers C., DeKlar M., Single leg lateral, horizontal, and vertical jump assessment: reliability, interrelationships and ability to predict sprint and change-of direction performance, J. Strength Cond. Res., 2009, 23 (4), 1140-1147, DOI: 10.1519/JSC.0b013e318190f9c2.

[15] Nagano A., Komura T., FuKashiro S., Optimal coordination of maximal-effort horizontal and vertical jump motions - a computer simulation study, Biomed. Engin. Online, 2007, 6 (20), 1-9, DOI: 10.1186/1475-925X-6-20.

[16] Paterno M., Ford K., Myer G., Heyl, R., Hewett T., Limb asymmetries in landing and jumping 2 years following anterior cruciate ligament reconstruction, Clin. J. Sports Med., 2007, 17, 258-262, DOI: 10.1097/JSM.0b013e31804c77ea.

[17] Popowczak M., Rokita A., Świerzko K., Szczepan S., Michalski R., MaćKAŁa K., Are linear speed and jumping ability determinants of change of direction movements in young male soccer players?, J. Sports Sci. Med., 2019, 18, 109-117.

[18] RodríGUEZ-LORENZo L., FERNÁNDEZ-Del Olmo M., SÁNCHEZ-Molin J.A., MARTÍN-ACERO R., Kicking ability and kicking deficit in young elite soccer players, Kinesiology, 2018, 50 (2), 194-203, DOI: 10.26582/k.50.2.2.

[19] RodríGuez-Lorenzo L., FernandeZ-Del-Olmo M., MartíN-ACERO R., Strength and kicking performance in soccer: A review, Strength Cond. J., 2016, 38 (3), 106-116, DOI: 10.1519 /SSC.0000000000000223.

[20] Rouissi M., Chtara M., Owen A., ChaAlali A., Chaouachi A., GABBETT T., Effect of leg dominance on change of direction ability amongst young elite soccer players, J. Sports Sci., 2016, 34 (6), 542-548, DOI: 10.1080/02640414.2015.1129432.

[21] RutKowska-Kucharska A., SzPala A., The use of electromyography and magnetic resonance imaging to evaluate a core strengthening exercise programme, J. Back Musculoskelet. Rehabil., 2018, 31, 355-362, DOI: 10.3233/ BMR-169780.

[22] Schiltz M., Lehance C., Maquet D., Bury T., CrierlaArd J., CROISIER J., Explosive strength imbalances in professional basketball players, J. Athl. Train., 2009, 44, 39-47.

[23] Śliwowski R., GRYGorowicz M., WieCZOREK A., JADCZAK Ł., The relationship between jumping performance, isokinetic strength and dynamic postural control in elite youth soccer player, J. Sports Med. Phys. Fitness, 2019, 58(9), 12261233, DOI: 10.23736/S0022-4707.17.07289-9. Epub. 2017, Jun 21.

[24] Turner A., Walker S., Stembridge M., Coneyworth P., ReEd G., Birdsey L., BARTer P., MoOdy J., A testing battery for the assessment of fitness in soccer players, Strength Cond. J., 2011, 33 (5), 29-39.

[25] Yanci J., Arcos A.L., Mendiguchia J., Brugheei M., Relationships between sprinting, agility, one- and two-leg vertical and horizontal jump in soccer players, Kinesiology, 2014, 46 (2), 194-201.

[26] ZAKAS A., Bilateral isokinetic peak torque of quadriceps and hamstring muscles in professional soccer players with dominance on one or both two sides, J. Sports Med. Phys. Fitness, 2006, 46 (1), 28-35. 